\title{
Severe brachial plexus injury after retropubic radical prostatectomy
}

\section{-A case report-}

\author{
Jaegyok Song
}

Department of Anesthesiology and Pain Medicine, Dankook University College of Medicine, Cheonan, Korea

A 69 -year-old man with prostate cancer underwent surgery for $16 \mathrm{~h}$. Approximately $6 \mathrm{~h}$ after surgery, the patient developed severe pain and motor weakness in his right arm. After neurologic examinations that included a nerve conduction study and electromyography, the patient was diagnosed with a brachial plexus injury. The causes of the brachial plexus injury were thought to be abduction of both arms, direct compression of the shoulder brace, and prolonged surgery. Most of the postoperative peripheral nerve injuries due to patient position are preventable, and anesthetists and surgeons should be very careful in positioning the patient accurately. (Korean J Anesthesiol 2012; 63: 68-71)

Key Words: Brachial plexus injury, Head-down tilt, Patient positioning.

Brachial plexus injury is a well-known complication that is associated with anesthesia and surgery $[1,2]$. Its mechanisms are unclear, but known contributing factors are the following: abduction of the arm more than $90^{\circ}$ (hands-up position), a low elbow level, shoulder braces with head-down position, diabetes mellitus, low-body temperature, and a longer duration of the disability $[1,3]$. Brachial plexus injuries typically exhibit relatively good prognosis and resolve within 10 weeks [4]. We report on a case with a postoperative, right-side brachial plexus injury with poor prognosis, despite the provision of definitive care, after a retropubic radical prostatectomy.

\section{Case Report}

A 69-year-old man (height, $170 \mathrm{~cm}$; weight, $85 \mathrm{~kg}$ ) with prostate cancer underwent a retropubic radical prostatectomy. Preoperatively, he had a history of taking medications for bronchial asthma, and laboratory findings were within normal limits.

Anesthesia was induced and maintained with propofol, rocuronium, fentanyl, and sevoflurane. The patient was placed in the lithotomy position with his arms abducted to $90^{\circ}$ on softpad-covered arm boards, and they were fixed loosely with cloth bands. Soft-pad-covered shoulder braces were placed over the

Received: April 11, 2011. Revised: 1st, June 6, 2011; 2nd, July 8, 2011; 3rd, July 23, 2011; 4th, August 4, 2011. Accepted: August 12, 2011.

Corresponding author: Jaegyok Song, M.D., Department of Anesthesiology and Pain Medicine, Dankook University College of Medicine, 16-5, Anseo-dong, Dongnam-gu, Cheonan 330-715, Korea. Tel: 82-41-550-6819, Fax: 82-41-550-6819, E-mail: drjack@nate.com

(C) This is an open-access article distributed under the terms of the Creative Commons Attribution Non-Commercial License (http:// creativecommons.org/licenses/by-nc/3.0/), which permits unrestricted non-commercial use, distribution, and reproduction in any medium, provided the original work is properly cited. 
coraco-clavicular joints. Cannulations were done uneventfully in the upper limbs, right internal jugular vein, and right radial artery. We used fluid warmer (Automer ${ }^{\circledR}$, Ace Medical, Seoul, Korea) and warm respiration circuit (Heated circuit ${ }^{\circledR}$, Ace Medical, Seoul, Korea) to prevent hypothermia during the surgery.

Because of the patient's obesity and massive adhesion, the operation time was $14 \mathrm{~h}$ and $40 \mathrm{~min}$, and anesthesia time was $16 \mathrm{~h}$ and $30 \mathrm{~min}$. The patient's position was lithotomy with Trendelenburg position (about $20-30^{\circ}$ ) during the surgery. Patient position was returned to level position every 4 hours for 10 to 15 minutes. The patient was hemodynamically stable during the anesthesia. There was no significant hypoxemia, hypothermia, hypovolemia and metabolic acidosis (Table 1). Blood loss was estimated around $800 \mathrm{ml}$, and urine output was not monitored due to the type of surgery. The total infused volume was $6 \%$ hydroxyethyl starch $2,000 \mathrm{ml}$, normal saline $3,000 \mathrm{ml}$, and lactated Ringer's solution 2,900 ml. Two units of packed red blood cells and four units of fresh frozen plasma were also infused.

After surgery, the patient recovered uneventfully and transferred to the intensive care unit. The patient had moderate facial edema but there was no respiratory difficulty after extubation. The patient had a $3 \times 4 \mathrm{~cm}$ sized, elevated skin lesion on his right shoulder and a $1 \times 2 \mathrm{~cm}$ sized, lesion on his left side. After $6 \mathrm{~h}$ in the intensive care unit, the patient complained of numbness with motor weakness in his right hand and forearm. He had flaccid paralysis in his forearm and hand, elbow and wrist flexion/extension were grade 2, shoulder abduction/adduction were grade 2 , and hypoesthesia was observed in the whole radial and median nerve site. On the sixth postoperative day (POD 6), his brachial plexus injury showed no improvement, and the pain with tingling sensation was increased. Physical treatments included arm elevation, transcutaneous electrical nerve stimulation, and hot packs that were continued for two weeks. On POD 20, the motor function of his right arm mildly improved, right shoulder abduction/ flexion was grade $3 / 2$, right elbow flexion/extension was grade $1 / 3$, first and second finger abduction/flexion was grade $1 / 2$, and right fingertip dysthesia was positive.

A neurological examination was performed on POD22. The sensory nerve conduction study showed no response in the right median nerve, the superficial radial nerve, and the medial/lateral antebrachial cutaneous nerve stimulation. The motor nerve conduction study showed decreased amplitude in the right median (wrist, elbow level), the ulnar (wrist, below elbow, above elbow), the radial, and the axillary nerves. In the musculocutaneous nerve, the amplitude was only 0 to 0.1 $\mathrm{mV}$. Needle electromyography showed no activity in the right biceps brachi, the triceps brachi, and the flexor carpi radialis muscles. The result was right brachial plexus injury with whole arm type-neuropraxic state or early partial axonotmesis. The patient was transferred to the rehabilitation department, and physical therapy was continued. However, the motor function of his right forearm did not recover, and his arm showed localized hyperthermia on the right, dorsal aspect of his hand with thermography, which estimates complex regional pain syndrome. On POD 38, the patient was discharged, and physical therapy was continued at the outpatient department. A followup neurological examination was performed on the fifth visit

Table 1. Patient's Vital Signs and ABGA Findings during the Surgery

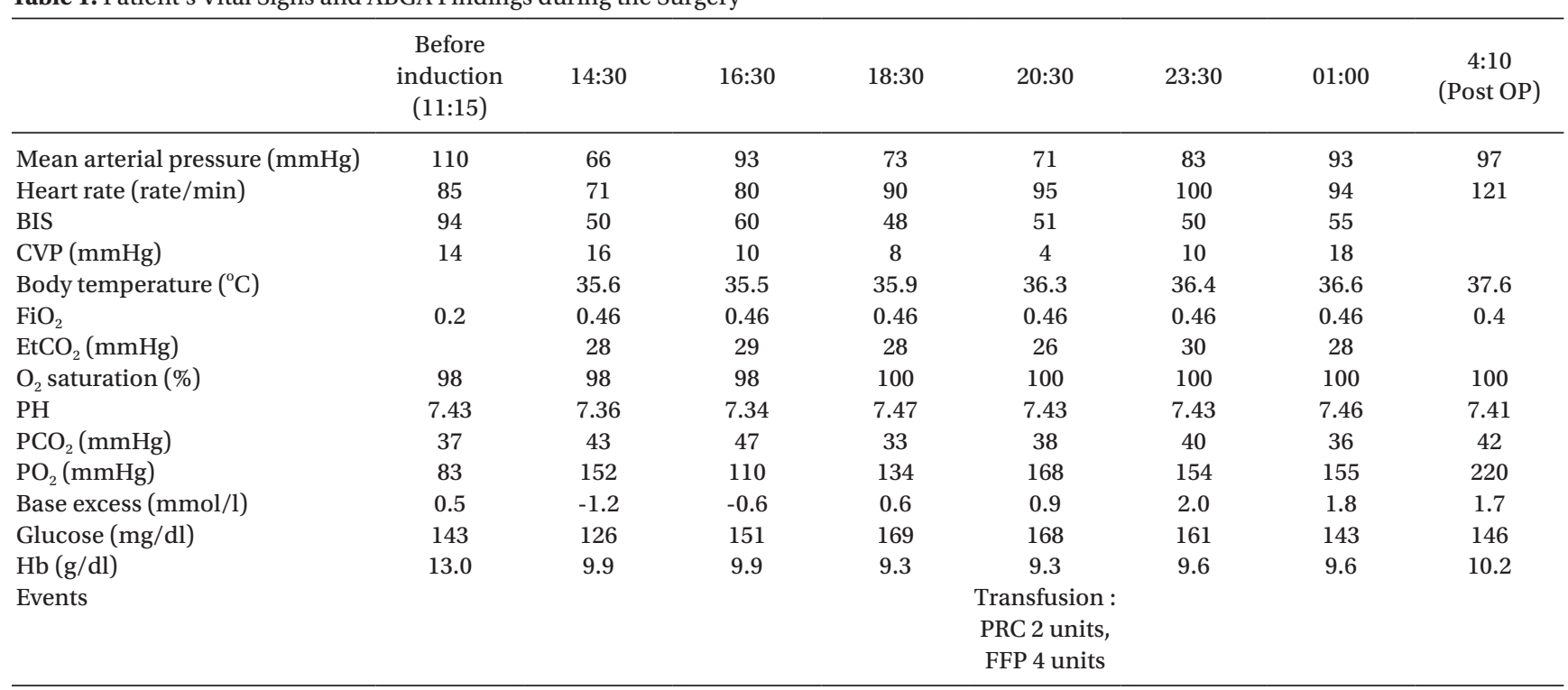


to the rehabilitation outpatient department after discharge (20 weeks after surgery), and his right shoulder abduction was grade 3 , his elbow flexion/extension grade was $0 / 3$, his wrist ventral flexion/dorsiflexion was grade $3 / 2$, and it was otherwise unremarkable. A sensory and motor nerve conduction study and electromyography were also performed, and the result was an incompletely recovered state in the axillary branch while other nerves were in a stationary state.

\section{Discussion}

Brachial plexus injury may occur after an abdominal operation due to the patient position on the operating table with the arms restrained on a board in abduction or hyperabduction, external rotation and dorsal extension of the arm, and flexion of the head to the contralateral side [4]. These positions can induce a stretch injury of the brachial plexus. Another mechanism is compression of the plexus by the head of the humerus, the first rib, and the clavicle [5].

In the 83 brachial plexus injuries that were reported between 1990 and 1995, eight cases were obviously related to intraoperative patient positioning. Four of these cases were due to the use of shoulder braces in the head-down position, three cases were due to improper positioning of the arm, and one case was due to sustained neck extension [2]. Hida et al. [1] suggested that relatively frequent neurologic injuries during liver transplantation, including brachial plexus injury, were probably related to prolonged anesthesia, invasive central venous monitoring catheters, and surgical procedures such as venoveno bypass and rib cage retraction. Even though we are aware of the risk of brachial plexus injury and we take precautions, these nerve injuries can still occur despite adequate padding of the affected nerve [6].

The patient's arms were positioned in $90^{\circ}$ abduction with shoulder braces during the surgery. Jackson and Keats [3] reported that there was no noticeable effect when the arm was abducted to $90^{\circ}$. However, Hida et al. [1] reported severe brachial plexus injury even when their patient's arms were abducted to $90^{\circ}$, which was considered safe. Michaels and Keats [7] reported three brachial plexus injuries after 113 consecutive radical retropubic prostatectomy cases with the arms secured laterally on arm boards. The investigators then began securing the patients' arms across their chests and reported no peripheral neuropathies after 127 cases [7].

We used shoulder braces and the Trendelenburg position during the surgery. Shoulder braces were used to prevent cephalad migration of the patient; however, the resultant pressure on the upper roots and trunks of the brachial plexus resulted in injuries to the brachial plexus [6]. Anatomic studies have demonstrated that braces can result in substantial com- pression and stretching of the brachial plexus $[5,8]$. Coppiesters et al. [9] strongly suggested that shoulder abduction and lateral rotation should be kept to a minimum and should never be combined with the use of shoulder braces. When shoulder braces were added, all tests resulted in a significantly reduced range of motion, even when the fixation force was applied over the advocated region like the acromio-clavicular joint. They recommended using a nonsliding mattress, regular control of the pressure on the shoulder girdle, and adjusting the position of the brace during anesthesia. We reversed the patient's Trendelenburg position four times, for 10 to 15 minutes each time, but it did not prevent his nerve injury.

We were not aware of the risk when shoulder braces are combined with shoulder abduction. Kent and Cheney [10] reported severe upper trunk brachial plexopathy after the use of shoulder braces with abducted arm position. They experienced decreased perfusion in both arms that was enough to produce a decreased pulse oximetry wave, fluctuation of blood pressure, and decreased radial artery pressure. The braces had been in place for $90 \mathrm{~min}$, and blood pressure returned to normal after the shoulder brace removal. Like our case, they were not aware of the risk of shoulder braces. But we did not experience any symptom or sign related to decreased circulation. Surveys by the ASA Task Force on the Prevention of Perioperative Peripheral Neuropathies reported that $34 \%$ of consultants and ASA members either disagreed or did not know that the use of shoulder braces might impact the risk of brachial plexus injury [10].

Gainsburg et al. [11] reported that there was no clinically significant brachial plexus injury in 575 cases of radical prostatectomy. They used horseshoe-shaped shoulder braces on patients who weighed $>75 \mathrm{~kg}$. They needed a steep Trendelenburg position $\left(45^{\circ}\right)$ to do robotic surgery, but their operation time was from 60 to $270 \mathrm{~min}$, and in open surgery, it was from 125 to $480 \mathrm{~min}$. Thus, we can assume that careful apply of horseshoe-shaped shoulder braces can be safe if surgery time is shorter than $8 \mathrm{~h}$.

Hida et al. [1] also reported a severe bilateral brachial plexus injury after liver transplantation that lasted for $16 \mathrm{~h}$. They did not use shoulder braces, but there was a severe bilateral brachial plexus injury that was caused by arm abduction. They concluded that slightly excessive stretching of the nerves for a long time caused the injury. In our case, the patient's injury was only on his right side. In addition, he had a bigger skin lesion on his right shoulder just after the end of the surgery. We assume that the pressure on his right shoulder was greater than that on his left side. It is thought that possibly the right shoulder brace was slightly advanced more than the left shoulder brace. Generally, this would be no problem, but the patient's obesity, the steep Trendelenburg position, and the longer duration of 
the surgery may have worsened the problem.

In order to prevent brachial plexus injury, the head should remain in the midline position, the arm should be kept at the side, the elbow should be gently fixed to unload the median nerve, and the forearm should be supinated in order to protect the ulnar nerve [4]. The wrist should be positioned in the neutral position, and elbow extension should be avoided as long as it is possible [4]. An excessive degree of the Trendelenburg position with shoulder braces should be avoided in order to decrease the pressure on the patient's shoulder. If it is not avoidable, care should be taken in applying the shoulder brace with appropriate padding and consider the use of a non-sliding mattress.

In conclusion, we experienced a patient with severe, right brachial plexus injury after retropubic radical prostatectomy due to arm abduction, shoulder braces and a very long duration of surgery with Trendelenburg position. Careful positioning of the upper extremity can decrease this kind of neurologic injury and disability. Especially when a longer duration of surgery is anticipated, care should be taken to lower the risk of peripheral nerve injury.

\section{References}

1. Hida A, Arai T, Nakanishi K, Nagaro T. Bilateral brachial plexus injury after liver transplantation. J Anesth 2008; 22: 308-11.

2. Cheney FW, Domino KB, Caplan RA, Posner KL. Nerve injury associated with anesthesia: a closed claims analysis. Anesthesiology 1999; 90: 1062-9.

3. Jackson L, Keats AS. Mechanism of brachial plexus palsy following anesthesia. Anesthesiology 1965; 26: 190-4.

4. Brill S, Walfisch S. Brachial plexus injury as a complication after colorectal surgery. Tech Coloproctol 2005; 9: 139-41.

5. Akhavan A, Gainsburg DM, Stock JA. Complications associated with patient positioning in urologic surgery. Urology 2010; 76: 130916.

6. Winfree CJ, Kline DG. Intraoperative positioning nerve injuries. Surg Neurol 2005; 63: 5-18.

7. Michaels MJ, Lish MC, Mohler JL. Patient positioning for radical retropubic prostatectomy. Urology 1998; 51: 824-6.

8. Coppieters MW. Shoulder restraints as a potential cause for stretch neuropathies: biomechanical support for the impact of shoulder girdle depression and arm abduction on nerve strain. Anesthesiology 2006; 104: 1351-2.

9. Coppieters MW, Van de Velde M, Stappaerts KH. Positioning in anesthesiology: toward a better understanding of stretch-induced perioperative neuropathies. Anesthesiology 2002; 97: 75-81.

10. Kent CD, Cheney FW. A case of bilateral brachial plexus palsy due to shoulder braces. J Clin Anesth 2007; 19: 482-4.

11. Gainsburg DM, Wax D, Reich DL, Carlucci JR, Samadi DB. Intraoperative management of robotic-assisted versus open radical prostatectomy. JSLS 2010; 14: 1-5. 